\title{
Un système d'imagerie 3-D
}

\author{
N. CESBRON, P. ALAIS, P. CHALLANDE et F. OLLIVIER
}

Laboratoire de Mécanique Physique et Expérimentale, Université Pierre et Marie Curie Paris 6, URA 879 du CNRS, 2 place de la Gare de Ceinture, 78210 St-Cyr-l'Ecole, France

\begin{abstract}
Within the EUROMAR 406 project, we have designed an acoustic camera that uses 2 orthogonal linear arrays. Site and azimuth scanning, at transmit and receive respectively, provides tridimensional data of the aquatic medium. The technology and morphology of the arrays are derived from a study on cylindrical antennas. Each array is $256 \mathrm{~mm}$ long and is made of 64 elements working at $500 \mathrm{kHz}$. These antennas are able to scan a $30^{\circ} \times 30^{\circ}$ sector with a $0.75^{\circ}$ resolution. The grating lobes artefacts are rejected under $-35 \mathrm{~dB}$. A fast dedicated numerical electronic system provides sectorial images in quasi-real time for ranges up to $100 \mathrm{~m}$. The transmit fan beams scan the $30^{\circ}$ wide sector according to 64 site directions and are wide open in the associated azimuth planes. Moreover they can be focused according to 32 finite distances. The 64 received signals undergo a continuous complex demodulation. A complex numerical computation (for 64 azimuths and 32 focal zones) over the 128 demodulated signals provides the sectorial image of the illuminated site at a rate of $2 \mu$ s per pixel. The 3-D sector is acquired after $\mathrm{N}$ site shots $(\mathrm{N}<64)$ providing as many sectorial images. For example, with $N=32,1 \mathrm{~s}$ is needed to collect the domain between $9 \mathrm{~m}$ and $12 \mathrm{~m}$ range. In association with a microcomputer the system can provide front views. A frontal imaging mode may be obtained in real time by opening the transmit beam in both site and azimuth
\end{abstract}

En vue de la recherche d'épaves, ou de la construction et de l'inspection de structures sous marines, nous avons développé dans le cadre du projet EUROMAR 406, une caméra acoustique permettant une imagerie 3D en temps réel. Construite à partir de deux réseaux orthogonaux $[1,2]$ de 64 éléments, la caméra fournit une image sectorielle de 64 rayons pour chaque site illuminé. Après avoir rappelé les performances des antennes que nous avons construites, nous exposerons notre technique de focalisation du faisceau d'émission, ainsi que le mode de reconstruction numérique d'une image sectorielle à partir des 64 signaux de réception. Enfin nous présenterons des images en élévation acquises en quasi temps réel de manière inédite.

\section{CARACTERISTIQUES ET PERFORMANCES DES ANTENNES.}

Les antennes d'émission et de réception sont identiques. Elles sont réalisées à partir de céramiques PZT fragmenté inclus dans une matrice de polymère. L'étude que nous avons menée [3] sur les antennes cylindriques s'est concrétisée par la construction de deux réseaux de 64 transducteurs au pas de $4 \mathrm{~mm}$ ayant à $500 \mathrm{kHz}$ une ouverture sélective de $30^{\circ} \times 30^{\circ}$ (cf. les diagrammes de directivité figures 1) permettant de réduire le niveau des artefacts d'image (dus aux lobes de périodicité) en deçà de $-35 \mathrm{~dB}$. 

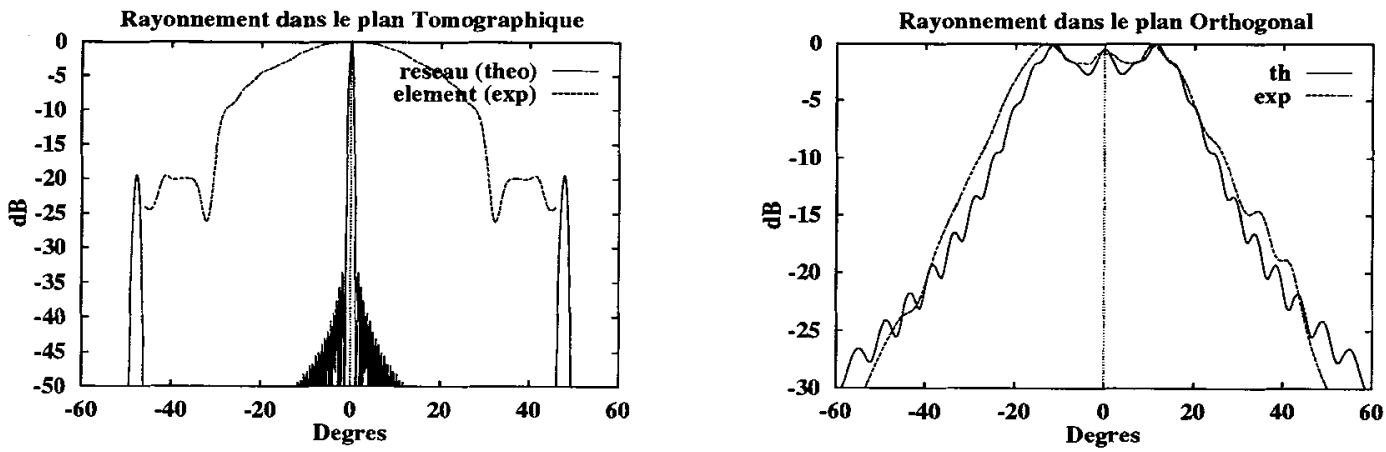

Figures 1: Diagrammes de directivité d'un élement de l'antenne.

\section{Focalisation a L'Emission}

2.1 Aussi bien en émission qu'en réception il est nécessaire de gérer correctement le déphasage de chaque élément afin de pouvoir focaliser en tout point du domaine d'exploration. Pour focaliser au point $P$ repéré en coordonnées polaires par $\mathbf{r}$ et $\boldsymbol{\theta}$, le déphasage associé au ième transducteur est lié à la différence de marche $r_{i}-\mathbf{r}$ (cf. fig. 2)

La focalisation électronique se fait de manière discrète pour 64 directions de l'espace (tous les demi-degrés), et pour $\mathbf{n}$ zones focales qui couvrent les portées de 1 à 200 mètres.

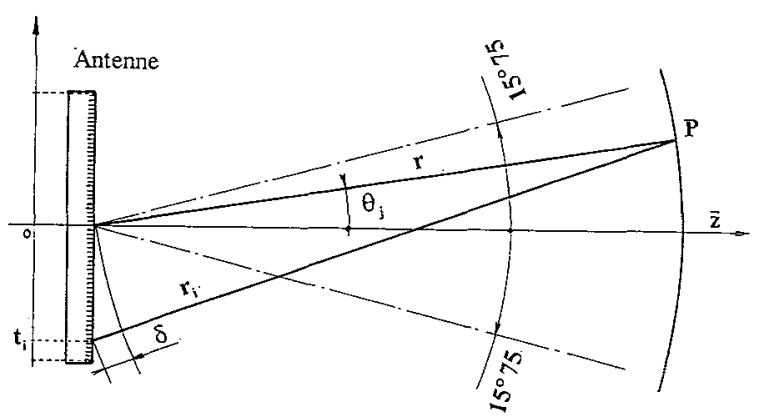

Fig. 2

Le nombre limité de zones focales introduit une différence entre le retard exact et retard calculé. Avec 32 zones focales, l'erreur maximale commise sur le déphasage reste inférieure à $2 \pi / 16$ (quelque soit la zone), ce qui relève le niveau des lobes secondaires de quelques pour-cent seulement. De la sorte, il est possible d'accommoder pour des portées allant de 1.3 à $200 \mathrm{~m}$.

Pour un site donné et une distance focale choisie, les 64 émetteurs sont activés par un signal harmonique correctement déphasé (fabriqué numériquement), modulé par une enveloppe parabolique dont la longueur dépend de la portée.

\subsection{Comparaison entre simulation et expérimentation.}

Les figures 3 présentent les diagrammes de rayonnement théoriques et expérimentaux, pour une émission focalisée à deux mètres pour les deux dépointages: $0.25^{\circ}, 12.25^{\circ}$.
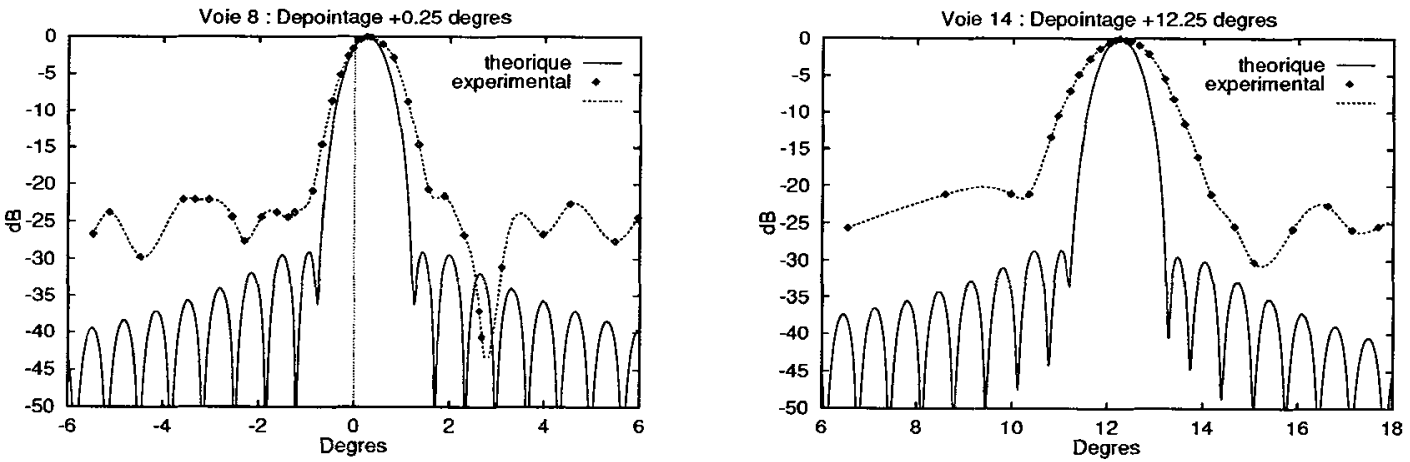

Figures 3: Diagrammes de rayonnement pour une émission focalisée à $2 \mathrm{~m}$. 
La direction de focalisation expérimentale correspond très correctement à la dirction visée. Cependant la résolution expérimentale est moins bonne que la résolution théorique, et cette différence s'accentue avec le dépointage.

La dégradation de la résolution provient de la courte durée de l'enveloppe. Le décalage temporel des enveloppes au point focalisé devient (pour les courtes distances) non négligeable devant la durée de l'enveloppe. Ce qui ce traduit par une dégradation de la focalisation pour les courtes portées et surtout pour les forts dépointages. On ne peut pas non plus allonger la durée de l'enveloppe car elle influe sur la résolution longitudinale. Le niveau des lobes secondaires ne s'élève jamais au dessus de $-20 \mathrm{~dB}$, pour de plus grandes portées on obtiendra (pour les mêmes raisons que précédemment) de meilleures performances, la longueur de l'enveloppe étant proportionnelle à la portée.

\subsection{Fonctionnement en imageur frontal.}

La camera 3D peut fonctionner en imageur frontal rapide lorsque l'émission illumine en un seul tir tout le domaine d'inspection. D'autre part il est possible de développer un appareil simplifié dérivé de la caméra fonctionnant avec une seule antenne défocalisée en émission et focalisée en réception. Dans les deux cas il faut être capable d'émettre un faisceau ouvert en site et en gisement. L'une des ouvertures est donnée par la géométrie cylindrique du réseau, l'autre est obtenue par défocalisation c'est à dire en focalisant à une distance négative (ici de -0.3 mètre).

\section{TRANSFORMEE COMPLEXE NUMERIQUE.}

\subsection{Construction d'un pixel d'une image sectorielle.}

La focalisation à l'émission fixe le plan dans lequel sera formée l'image. Le signal émis est caractérisé par sa pulsation $\omega$ et son enveloppe temporelle $A(t)$, on peut le représenter par: $\mathbf{A}(\mathbf{t}) \cdot \mathbf{e}^{\mathrm{j} \omega t}$. On admet qu'un point échogène réémet une onde sphérique de la forme: $A(t-r / c) e^{j \omega(t-r / c)}$

Ce signal à bande étroite $(\mathbf{A}(\mathrm{t})$ enveloppe à variations limitées), atteint le ième récepteur à l'instant: $\mathbf{t}-\mathbf{r}_{\mathrm{i}} / \mathbf{c}$ (cf. figure 2). La contribution du ième transducteur au signal d'écho fourni par une cible ponctuelle sera à un facteur près (atténuations, réflexivité) : $S_{\mathbf{i}}(t)=A\left(t-r_{\mathbf{i}} / \mathbf{c}\right) \mathbf{e}^{\mathrm{J} \omega\left(t-r_{\mathbf{i}} / \mathbf{c}\right)}$

Les $64 \mathrm{~S}_{\mathrm{i}}(\mathbf{t})$ devront donner l'amplitude de l'onde acoustique émise par les points du secteur inspecté. Pour cela, deux méthodes sont classiquement utilisées pour compenser les retards $\mathbf{r}_{\mathbf{i}} / \mathbf{c}$. L'une consiste à retarder le signal reçu par chaque transducteur $\mathrm{Ti}$ de $\mathbf{r}_{\mathbf{i}} / \mathbf{c}$ (à une constante temporelle près). L'autre consiste à compenser les déphasages. Pour les dispositifs d'imagerie à haute fréquence, les retards correspondent à plusieurs longueurs d'onde, seule la remise en phase est envisageable dans le cadre d'un traitement numérique. Les problèmes induits par l'échantillonnage accompagnant nécessairement un traitement numérique des signaux hautes fréquences sont partiellement résolus en opérant une double détection synchrone en quadrature avec une fréquence de référence identique à celle d'émission. Les signaux hétérodynés sont échantillonnés au quart de la durée du signal émis. Ce qui suffit pour obtenir une résolution longitudinale de qualité. Chaque échantillon subit une double correction :

1) un recalage temporel (grossier) relatif au parcours point image / transducteur concerné.

2) une compensation de phase (fine) par multiplication complexe par le terme $: e^{j \omega\left(+r_{1} / c\right)}$.

Le produit obtenu est la contribution élémentaire d'un transducteur Ti au signal d'écho pour le point considéré de l'image. La sommation des 64 contributions correctement retardées et déphasées donne le signal d'écho au point cherché. Les 128 signaux démodulés sont multiplexés 16 par 16 puis numérisés avant d'être stockés dans huit RAMs. Le recalage temporel se fait avec une précision $\pm 1 / 8$ de la durée de l'enveloppe (induisant une erreur sur la somme des contributions inférieure à $5 \%$ ) au moment de la lecture des RAMs. La compensation de phase s'opère ensuite par multiplication avec les parties réelles et imaginaires du terme de remise en phase approximé (comme en émission focalisée) avec les 32 zones focales, et pour 64 azimuts. Le calcul d'un pixel requiert $2 \mu \mathrm{s}$, une image sectorielle complète d'une profondeur de 512 points est calculée en $64 \mathrm{~ms}$.

\subsection{Résultats expérimentaux de la transformée complexe.}

En déplaçant dans un plan tomogaphique situé à $2 \mathrm{~m}$ de l'antenne de réception, un petit émetteur peu directif, nous avons relevé les diagrammes de rayonnement issus de la transformation complexe. Pour des 
dépointages de $-9.75^{\circ}$ et $0.25^{\circ}$, nous comparons en figures 4 les diagrammes de focalisation théoriques et ceux obtenus par la transformation complexe.
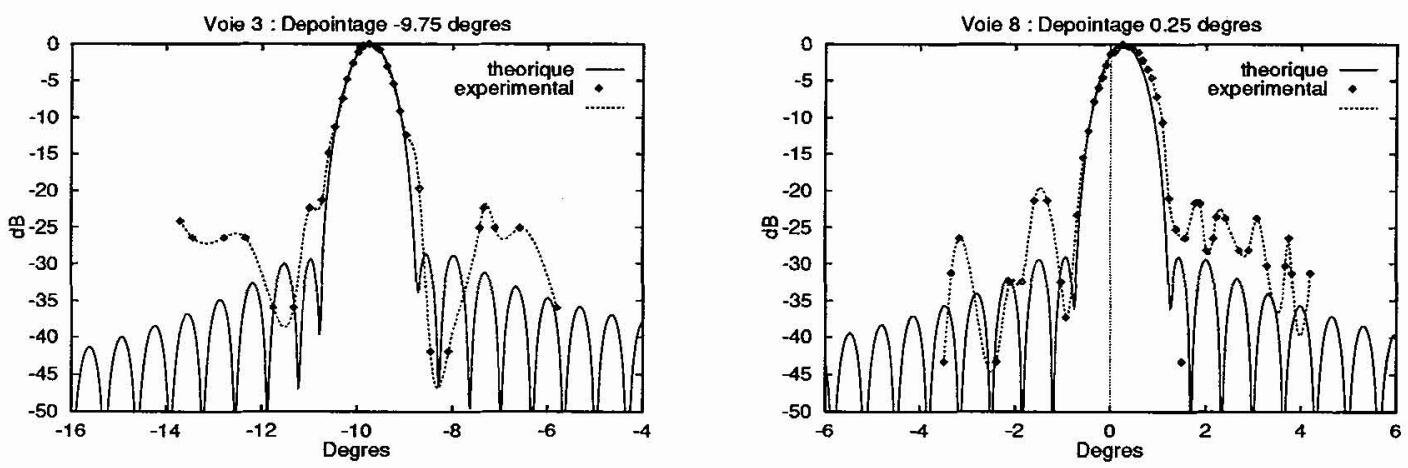

Figures 4: Diagramme de focalisation en réception

Les diagrammes théoriques et expérimentaux concordent beaucoup mieux qu'à l'émission. On peut de ce fait mesurer l'impact du recalage temporel des échantillons sur la qualité de la focalisation. Si l'on veut améliorer la sélectivité en émission, il faut retarder les enveloppes en fonction du transducteur, de la focale et du dépointage concernés. D'autre part les niveaux des lobes secondaires et de périodicité ne dépassent pas les $-20 \mathrm{~dB}$, vu la sélectivité du digramme de rayonnement dans le plan orthogonal (cf. figures 1), l'artéfact lié au lobe de réseau possédera un niveau inférieur à $-40 \mathrm{~dB}$.

\section{LES IMAGES OBTENUES.}

Pour un site choisi, l'image sectorielle calculée en $64 \mathrm{~ms}$ apparaît sur un écran vidéo. Un mode automatique de balayage en site saisit le domaine $3 \mathrm{D}$, le défilement des coupes tomographiques à l'écran permet au cerveau de recomposer une perspective. $L^{\prime}$ image en élévation (fabriquée lors du mode automatique de balayage) correspondant à une tranche sélectionnée du domaine, apparaît sur le moniteur d'un micro ordinateur. Les figures suivantes présentent une mire de Siemens de diamètre $50 \mathrm{~cm}$ à huit rayons (figure 5),un élément de structure offshore (figure 6).
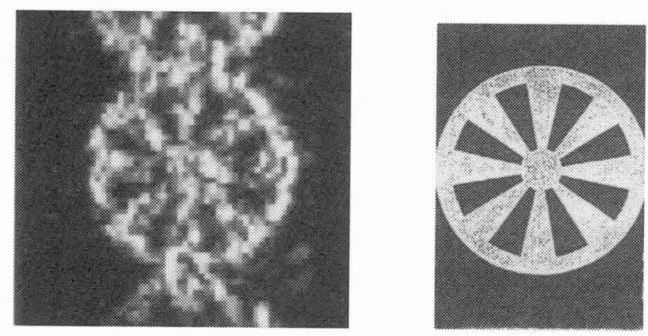

Figure 5: Mire de Siemens placée à $1.5 \mathrm{~m}$, vue avec ses réflexions sur la surface et le fond de l'aquarium.
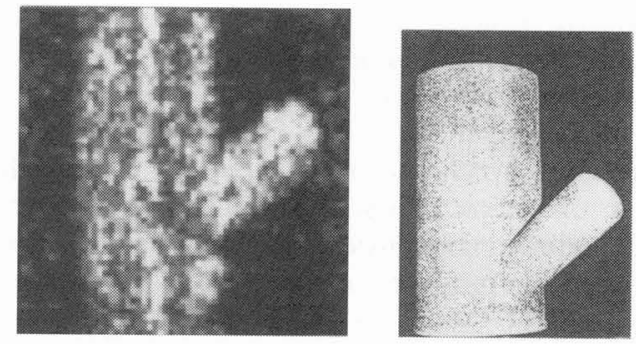

047 Figure 6: Elément de structure situé à $2 \mathrm{~m}$

\section{Références:}

[1] W.H. WELLS -Acoutical Holography, 2, p. 87 (1969).

[2] P.ALAIS, P.CHALLANDE."Developpement of a underwater frontal imaging sonar. Concept of a 3-D imaging system".In :"Acoustical Imaging",18, Plenum Press, Santa Barbara (1989).

[3] N.CESBRON,P.ALAIS. "A 3D acoustical imaging system".European conference on underwater acoustics, p. 756 (1992) 